\title{
The effect of syllabic stress and syllabic organization on the identification of speech sounds
}

\author{
LAURA L. HALL and SHEILA E. BLUMSTEIN \\ Brown University, Providence, Rhode Island 02912
}

\begin{abstract}
The present study was undertaken to investigate the effects of syllabic stress and segment structure on selective adaptation in speech. To this end, a CV place of articulation test continuum was selectively adapted by seven different adapting stimuli; the monosyllables [ba] and [ga], two disyllabic stimuli containing equal stress on both syllables, [baga] and [gaba], and three disyllabic stimuli ([baga]) in which stress placement varied and was cued by the acoustic parameters of fundamental frequency and duration. Results for the two adapting stimuli demonstrated significant [b] adaptation for the stimulus [ba] and significant [g] adaptation for [ga]. Of the five other adapting stimuli, only [g] adaptation for the stimulus [bagá] was found to be significant. These findings indicate that the operation of detector mechanisms susceptible to fatigue by an adapting stimulus are even more constrained than has heretofore been suggested. It appears that the adapting and test stimuli must not only have the same phonetic and syllable structure, but also the same syllabic organization.
\end{abstract}

Recent studies have suggested that speech perception may involve an analysis of complex stimuli by feature detectors into their component properties. The postulated psychological reality of feature detectors was initially inferred from data concerning perceptual confusions among phonemes (Miller \& Nicely, 1955; Singh, 1966), short-term memory loss for feature information (Wickelgren, 1965, 1966), and subjective judgments of phonemic similarity (Greenberg \& Jenkins, 1964). Neurophysiological evidence also exists that indicates the existence of analyzers that are sensitive to complex aspects of the acoustical signal (e.g., Evans \& Whitfield, 1964). Although these studies were concerned with psychological responses of cats to these complex auditory nonspeech stimuli, the results obtained have been used as indirect evidence for the potential existence of speech detector mechanisms for speech in humans.

The strongest evidence as to the nature of feature detectors has come from studies utilizing a selective adaptation procedure. This paradigm is based upon the theory that if feature detectors do exist for the analysis of stimulus patterns, then repeated stimulation of these structures should cause them to fatigue and alter their manner of functioning. They would thus be rendered less efficient or sensitive. Therefore, by altering the listeners' perception along any selected feature dimension, one can hope to obtain information about the perceptual system as a whole.

Eimas and Corbit (1973) utilized this selective adaptation procedure on a series of synthetic consonant-vowel (CV) syllables varied along the acoustic dimension of voice onset time (VOT). This acoustic cue serves in English to distinguish between the voiced stops $[b, d, g]$ and the voiceless stops $[p, t, k]$. The experimenters reasoned that if linguistic feature detectors mediate the perception of voiced and voiceless stops, then repeated presentation of stimuli having the shared feature of voicing should cause the detector of that feature to become fatigued and thus less effective. Results showed that if the adaptation stimulus was voiced, a greater number of identification responses were now of the unadapted, voiceless category. That is, adaptation with a voiced stimulus resulted in fewer identification responses in comparison to the functions obtained in the unadapted state. The reverse was also found to be true; a voiceless adapting stimulus resulted in more identification responses of the voiced category. The findings of the Eimas and Corbit study were significant in several respects. First, they indicated that a selective adaptation paradigm is effective in producing a change along a particular acoustic dimension corresponding to a single linguistic feature dimension. Second, the results supported the original hypothesis of the existence of linguistic feature detectors.

The type of information intrinsic to the speech signal necessary to operate these feature detectors has been the topic of serious debate in the recent literature. The particular topic in question is whether feature adaptation in fact operates on detectors that are sensitive to relatively invariant acoustic cues or whether these detectors are sensitive to feature information for which there may exist no corresponding invariant acoustic cue. Cooper (1974a) hoped to resolve this debate by testing the effects of adaptation on a continuum of speech 
sounds varying along the place of articulation feature dimension. In particular, he sought to determine whether the adaptation procedure will produce shifts in the loci of phonetic boundaries comparable to those found previously in the case of VOT (Eimas \& Corbit, 1973). It was reasoned that if adaptation affects linguistic feature detectors that operate on relatively invariant acoustic information, then no shifts should occur for the second- and thirdformant transition cues for place, since these acoustic cues vary greatly as a function of the vowel environment. Conversely, if adaptation affects some higher level mechanism that is specialized for extracting phonetic feature information, one would expect perceptual shifts for the place of articulation feature.

The test series consisted of synthetic CV syllables which varied in both direction of the second-formant and third-formant transitions. Significant bilabialalveolar [b-d] boundary shifts were obtained after adaptation with [b] and significant alveolar-velar [d-g] boundary shifts were obtained after adaptation with [g]. Acoustically different adapting stimuli such as [bi] also produced significant boundary shifts, although the shifts obtained for adapting stimuli with a different vowel were smaller than those where the vowel was not varied (Cooper, 1974b). These results suggested that adaptation for the place of articulation feature occurred mainly at a phonetic rather than at an acoustic level.

Other evidence suggesting feature detectors at a phonetic level has been reported by Diehl (1975). He showed that a burst-cued adapting stimulus moved the phonetic boundary for a place of articulation continuum containing transition cues only. Because the adapting and test stimuli shared the same phonetic cues but not the same acoustic cues, Diehl concluded that at least part of the adaptation effect occurs at a site of phonetic, and not merely acoustic, feature analysis.

The conclusion that the level of speech processing is at the phonetic rather than acoustic level has been seriously questioned by several studies. Cooper and Blumstein (1974) have demonstrated that it is not the phonetic dimension of the adapting stimulus but its acoustic structure that determines the direction of adaptation effects. Tartter and Eimas (1975) found that the greater the acoustic overlap between the adapting stimulus and the test continuum, the greater the adaptation effect. In fact, the results of Diehl's (1975) study could be interpreted in terms of a single acoustic feature detector which integrates both burst and transition information. Such a hypothesis has been given some support in a recent study (Blumstein, Stevens, \& Nigro, 1977). They showed that adaptation effects for a transition-cued place of articulation continuum were greater when the adapting stimulus contained both burst and transition information than when the adapting stimulus contained transition information only.

Ades (1974) has also seriously questioned a phonetic locus of the adaptation effects. He constructed two synthetic speech continua, one ranging from [bæ] to [dæ], the other form [æb] to [æd]. For both continua, only the F2 and F3 transitions varied. Since these two series are mirror images of each other, transitions which rise in the syllable [bæ] fall in the syllable [ab]. Ades reasoned that if the phonetic feature detector theory is correct, repetition of [bæ] should have a [b]-adapting effect on [æb/æd]. However, if the detectors are only tuned to the rising vs. falling patterns of frequency change, regardless of context, then repetition of [bæ] will adapt and thus weaken detectors for rising transitions and thereby [d]-adapt an [æb/æd] series.

Results of the experiment showed that if the adapting and test sounds were from the same series, stimuli containing a [b] produced [b]-adaptation and stimuli containing a [d] caused [d]-adaptation. However, when the adapting and test stimuli were from different continua, all four adapting stimuli gave a slightly [d]-adapting effect. These results led Ades to reject the phonetic theory of feature detection. He argued that a truly phonetic detector would not distinguish between initial and final environments. That is, feature detection should not be position sensitive. Ades was led to adopt a theory postulating the existence of different detectors for consonants in initial and final position.

In a later study (Pisoni \& Tash, 1975), it was found that a nonspeech VC stimulus produced shifts in the perception of a $\mathrm{CV}$ place test series. However, the shifts obtained were based upon the spectral similarity of the adaptor and test stimuli and not upon the phonetic characteristics of the stimuli, again suggesting that the adaptation of a phonetic feature seems to be position sensitive.

The present study is intended as an extension of the Ades study (1974) which investigated position sensitivity of the speech feature detectors. Ades has demonstrated that consonants in final position [æb/ad] fail to produce boundary shifts on a continuum of $\mathrm{CV}$ syllables. In the present study, the effects of two-syllable adapting stimuli (e.g., [baga]) on the perception of a $\mathrm{CV}$ continuum were assessed in order to determine whether adaptation shifts would be found for consonants in intervocalic position. That is, would the stimulus [baga] produce [g] adaptation? The formant transitions signaling the intervocalic consonants are not mirror images of those signaling consonants in the test continuum as in the Ades study. Rather, the formant transitions cuing consonants in both the test continuum and 
adapting stimuli are identical. The only difference between the two is that consonants of the test continuum are in stimulus-initial position as well as syllable-initial position while those of the adapting stimuli are solely in syllable-initial position. Thus, unlike the stimuli used in Ade's study, both the adapting and test stimuli share the same onset and formant structure characteristics. If, in the present study, the adapting stimulus [baga] produces significant [g]-adaptation as well as [b]-adaptation, then theories postulating a phonetic speech processor (e.g., Ades, 1974; Cooper, 1974a; Diehl, 1975) or detector mechanisms sensitive to onset characteristics would be supported. If, however, adaptation effects are dependent upon an isomorphism of the phonetic, i.e. syllable, structure, as well as acoustic fine structure between the adapting and test stimuli, then one would anticipate significant adaptation for the initial consonant but not necessarily for the intervocalic consonant of the adapting stimulus. The contextual effects of a preceding syllable may effectively eliminate the possibility for the adaptation effect to occur despite the fact that the intervocalic consonant is in syllable-initial position.

The prospective results outlined above are postulated without regard to syllabic stress within the adapting stimulus. As single-syllable stimuli carry main stress, it is impossible to titrate out any effect that this variable may have on adaptation effects. However, it is possible to examine the effect of this factor when dealing with two-syllable adapting stimuli. Stress may be signaled acoustically in several ways: by varying the fundamental frequency of the syllable, by altering vowel length, by modifying intensity, or a combination of these factors (Fry, 1958; Lehiste \& Peterson, 1959). A stressed syllable may fatigue the feature detectors to a greater extent than would an unstressed syllable. As a result, one may find significant boundary shifts for the intervocalic consonant when it receives primary stress, in contrast to when the syllable is unstressed.

\section{METHOD}

\section{Subjects}

Eight students attending Brown University served as subjects in this study. All were native speakers of English with no known hearing impairment. Five of the eight subjects had previous experience in hearing synthetic speech.

\section{Stimuli}

The test stimuli were a series of 14 synthetic $\mathrm{CV}$ syllables generated on the PDP-9 computer facility at the MIT Research Laboratory of Electronics, using a program written by D.H. Klatt (1972). The program simulated a parallel resonance synthesizer. The $14 \mathrm{CV}$ stimuli were five-formant syllables of an overall duration of $250 \mathrm{msec}$. Each had initial piecewise-linear transitions on the first three formants and a final steady-state formant corresponding to the vowel [a]. Steady-state formant frequencies (and bandwidths) for the vowel were centered at $720(50), 1,170(70), 2,600(90), 3,500(160)$, and 4,500 (250) $\mathrm{Hz}$. Stimuli differed from one another only in the starting frequency and direction of the second-formant and third-formant transitions. This provides sufficient cues for the perception of variation in place of articulation. The transitions for a typical stimulus are displayed in the upper panel of Figure 1. The durations of the Formant 2 and Formant 3 transitions were $40 \mathrm{msec}$ for all 14 stimuli. The starting frequencies of the secondand third-formant transitions at the stimulus onset are shown in Figure 2. The starting frequency of the first formant transition was $200 \mathrm{~Hz}$. The first formant transitions differed slightly in both duration and shape from one stimulus to another. The duration ranged from $30 \mathrm{msec}$ for Stimulus 1 to $55 \mathrm{msec}$ for Stimulus 14 . These changes were made to improve the quality of the percept for the best exemplars of [b], [d], and [g], and have been used in other adaptation experiments (see Blumstein, Stevens, \& Nigro, 1977). The fundamental frequency contour for the stimuli are displayed in the lower panel of Figure 1. The contour started at $103 \mathrm{~Hz}$ and rose piecewise linear to $125 \mathrm{~Hz}$ for the first $35 \mathrm{msec}$ of the stimulus, fell piecewise linear to $94 \mathrm{~Hz}$ during the next $180 \mathrm{msec}$, and then to $50 \mathrm{~Hz}$ for the last $70 \mathrm{msec}$ of the stimulus.

The adapting stimuli consisted of the seven synthetic syllables [ba], [ga], [baga], [gaba], [bága], [bagá], and [bagá] (lowered [ba] fundamental frequency). Two of these stimuli, [ba] and [ga], were identical to the 1st and 12th stimuli of the test continuum. Two others were constructed by combining these two singlesyllable stimuli to produce the two-syllable adapting stimuli [baga] and [gaba]. The two syllables comprising each stimulus were separated by $80 \mathrm{msec}$. This interval allowed for a clear perceptual separation of the syllables, yet the entire stimulus was still perceived as a single unit. Each syllable of these stimuli
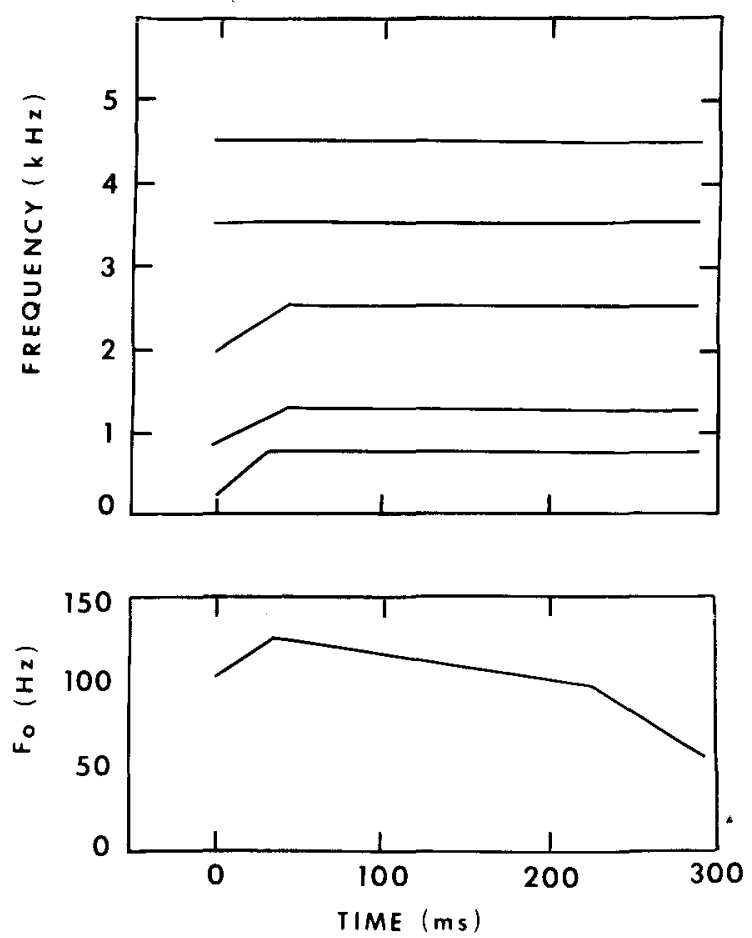

Figure 1. The upper panel displays the formant transitions for a typical stimulus used in this study. The lower panel illustrates the fundamental-frequency contour for the synthesizer excitation pulses. (After Blumstein, Stevens, \& Nigro, 1977.) 
received equal stress. The remaining three stimuli were twosyllable [baga] stimuli, in which stress was placed on either the first or second syllable. To this end the fundamental-frequency contour of the syllable to be stressed was raised $15 \mathrm{~Hz}$ and the length of the unstressed syllable was shortened by $45 \mathrm{msec}$. These alterations produced two adapting stimuli [bága] and [bagá]. Another two-syllable [bagá] adapting stimulus was produced by lowering the [ba] fundamental-frequency contour $15 \mathrm{~Hz}$ and keeping the length of both syllables the same.

\section{Procedure}

All eight subjects were tested in seven separate 1-h sessions. Each session included one baseline identification series and an adaptation series using one of the seven adapting stimuli. In order to make sure that all subjects began each session in an unadapted state, the experimental sessions were held at least $24 \mathrm{~h}$ apart. All stimuli were presented on an Ampex AG-500 tape recorder and a Crown 820-144 tape recorder via four matched sets of Koss Pro 600AA earphones.

A baseline identification function was obtained for all subjects prior to the commencement of each adaptation test. The identification series included 10 occurrences of each of 14 different stimuli presented in random order with an interstimulus interval of $3 \mathrm{sec}$. Four practice stimuli were included, two at the beginning of the test and the other two directly following a break which occurred after presentation of the first 72 stimuli. These trials were included in the actual listening tests but were not scored. Subjects were told to classify each stimulus as [ba], [da], or [ga] and to record their answer by writing the appropriate initial consonant on the answer sheet provided. The subjects were instructed to make their responses as soon as possible after hearing each identification stimulus.

Following the initial baseline series, identification functions were obtained for the same test series after selective adaptation with one of the seven adapting stimuli. In order to avoid any possible order effect, each subject received a different ordering of the adaptation stimuli. The adaptation test began with the repeated presentation of the adapting stimulus for $3 \mathrm{~min}$. Each repetition of the stimulus was separated by $350 \mathrm{msec}$ of silence. Subjects then listened to four stimuli from the identification series which they were asked to identify. As in the baseline series, they then wrote the appropriate initial consonant on the answer sheet provided.

After this initial adaptation period, 36 adaptation trials were administered. On each trial, the subjects listened to the adapting stimulus for $1 \mathrm{~min}$ followed by four randomly selected stimuli from the series of 14 stop consonants. Again, as in the baseline series, they identified the initial stop consonants and wrote them on the answer sheet provided. After the fourth stimulus was presented for identification, the subjects immediately began the next adaptation trial. The subjects were given a short break after the first 18 adaptation trials.

\section{RESULTS}

Mean identification functions across all eight subjects for the seven adaptation conditions are seen in Figures 3 and 4 . The solid lines represent the identification functions obtained in the unadapted state and the dotted lines represent the identification functions obtained after selective adaptation. Individual and mean phonetic boundary loci for each of the seven adaptation conditions are seen in Table 1. Boundary loci were computed using a least mean squares analysis on $z$-scores derived from the

Table 1

Individual and Mean Loci of Phonetic Boundaries for Each Test Condition

\begin{tabular}{|c|c|c|c|c|c|c|c|c|}
\hline \multirow[b]{2}{*}{ Subject } & \multirow[b]{2}{*}{ Baseline } & \multicolumn{7}{|c|}{ Adaptation with } \\
\hline & & [ba] & [ga] & [baga] & [gaba] & [bága] & [bagá] & [bagáa * \\
\hline \multicolumn{9}{|c|}{ [b-d] Phonetic Boundary } \\
\hline $\begin{array}{l}\text { J.C. } \\
\text { A.D. } \\
\text { A.F. } \\
\text { J.R. } \\
\text { R.L. } \\
\text { H.M. } \\
\text { E.Z. } \\
\text { V.T. }\end{array}$ & $\begin{array}{l}5.20 \\
5.50 \\
5.50 \\
5.50 \\
5.50 \\
5.61 \\
5.50 \\
5.50\end{array}$ & $\begin{array}{l}4.58 \\
4.50 \\
4.44 \\
5.12 \\
4.60 \\
5.12 \\
4.98 \\
3.76\end{array}$ & $\begin{array}{l}5.50 \\
5.12 \\
5.50 \\
5.50 \\
5.50 \\
6.00 \\
5.50 \\
5.50\end{array}$ & $\begin{array}{l}4.02 \\
5.08 \\
5.12 \\
5.50 \\
5.10 \\
5.88 \\
5.08 \\
5.02\end{array}$ & $\begin{array}{l}4.98 \\
5.50 \\
5.50 \\
5.50 \\
5.12 \\
5.50 \\
5.50 \\
5.50\end{array}$ & $\begin{array}{l}4.00 \\
5.50 \\
5.50 \\
5.88 \\
5.50 \\
5.50 \\
5.50 \\
5.02\end{array}$ & $\begin{array}{l}4.92 \\
5.50 \\
5.12 \\
5.88 \\
5.08 \\
5.50 \\
5.50 \\
5.05\end{array}$ & $\begin{array}{l}4.12 \\
4.98 \\
5.50 \\
5.88 \\
5.30 \\
5.50 \\
5.12 \\
4.95\end{array}$ \\
\hline Mean & 5.48 & 4.64 & 5.52 & 5.09 & 5.39 & 5.30 & 5.32 & 5.67 \\
\hline \multicolumn{9}{|c|}{ [d-g] Phonetic Boundary } \\
\hline $\begin{array}{l}\text { J.C. } \\
\text { A.D. } \\
\text { A.F. } \\
\text { J.R. } \\
\text { R.L. } \\
\text { H.M. } \\
\text { E.Z. } \\
\text { V.T. }\end{array}$ & $\begin{array}{l}10.72 \\
10.43 \\
10.44 \\
10.60 \\
11.28 \\
10.44 \\
10.81 \\
10.66\end{array}$ & $\begin{array}{l}10.50 \\
10.50 \\
10.56 \\
10.71 \\
10.98 \\
11.00 \\
10.98 \\
11.05\end{array}$ & $\begin{array}{l}13.56 \\
11.66 \\
11.02 \\
11.14 \\
11.99 \\
12.27 \\
11.50 \\
11.79\end{array}$ & $\begin{array}{r}12.27 \\
10.88 \\
11.08 \\
11.05 \\
10.92 \\
9.88 \\
11.00 \\
10.50\end{array}$ & $\begin{array}{l}10.50 \\
10.26 \\
10.88 \\
11.00 \\
11.11 \\
10.58 \\
10.88 \\
10.92\end{array}$ & $\begin{array}{l}10.98 \\
10.92 \\
11.16 \\
10.95 \\
11.04 \\
10.92 \\
11.05 \\
11.08\end{array}$ & $\begin{array}{r}12.19 \\
10.92 \\
11.16 \\
9.62 \\
12.57 \\
10.98 \\
11.02 \\
11.92\end{array}$ & $\begin{array}{l}10.50 \\
10.08 \\
10.50 \\
10.92 \\
11.05 \\
10.50 \\
10.50 \\
10.50\end{array}$ \\
\hline Mean & 10.67 & 10.79 & 11.87 & 11.09 & 10.77 & 11.01 & 11.30 & 10.57 \\
\hline
\end{tabular}

Note-The loci of the phonetic boundaries for the "baseline" (i.e., without adaptation) condition displayed here are the averages of all seven baseline functions obtained prior to each adaptation testing.

*Lowered [ba] fundamental frequency. 
percentages of identification responses assigned to each stimulus. These assigned numbers correspond to the stimulus numbers assigned in Figure 2.

Since no comparisons were to be made between data for [b] adaptaton and data for [g] adaptation, these two groups of data were analyzed separately. A repeated measures two-factor analysis of variance (baseline/adaptation by adapting condition) was performed on each group of data. Significant main effects for [b]-adaptation data are seen for both adaptation $[\mathrm{F}(1,7)=11.72, \mathrm{p}<.05]$ and adapting condition $[F(6,42)=5.84, p<.01]$. A significant main adaptation effect is also seen for [g]-adaptation data $[F(1,7)=19.89, \quad p<.01]$, while a nonsignificant main effect for adapting condition is obtained $[F(6,42)=.437, p>.20]$.

Subsequent post hoc comparisons (Duncan test) were performed to determine if there were significant shifts in the loci of the phonetic boundary relative to the baseline condition for each adaptation condition. These results are seen in Table 2 . Results indicate highly significant [b]-adaptation for the

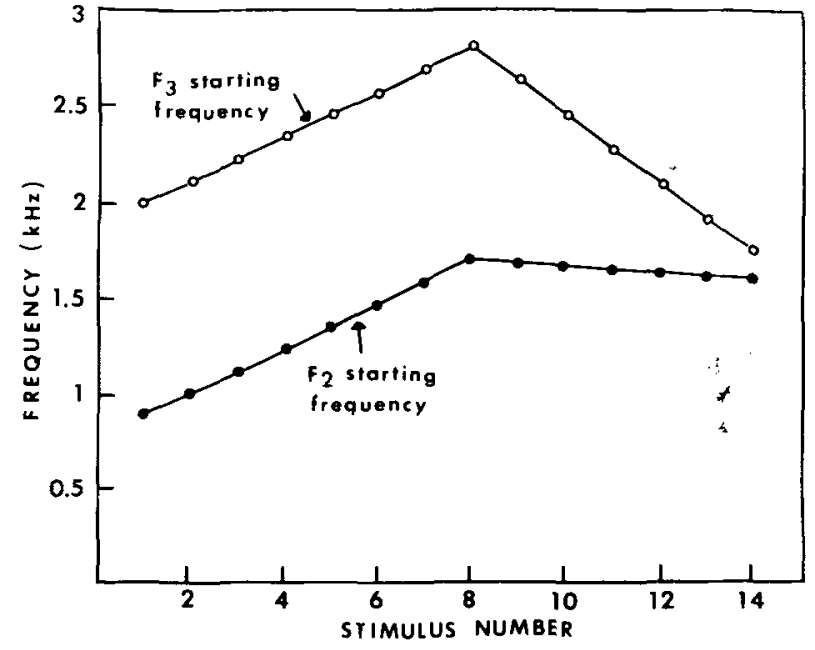

Figure 2. Starting frequencies for the second-formant and thirdformant transitions at the stimulus onset. (After Blumstein et al., 1977.)
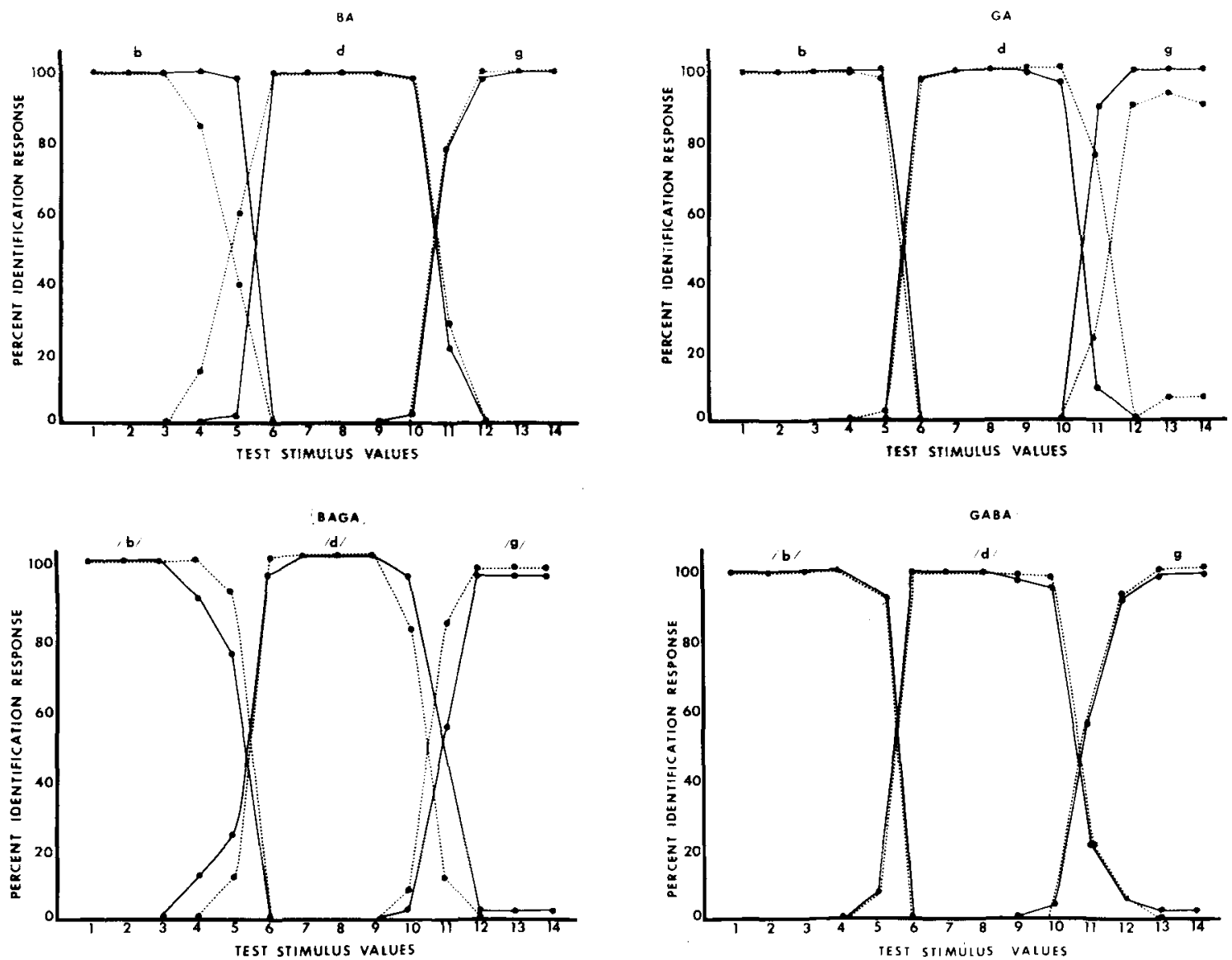

Figure 3. Mean identification functions across eight subjects for the seven different adapting conditions. The solid lines represent the identification functions obtained in the unadapted state, and the dotted lines represent the functions obtained after selective adaptation. 

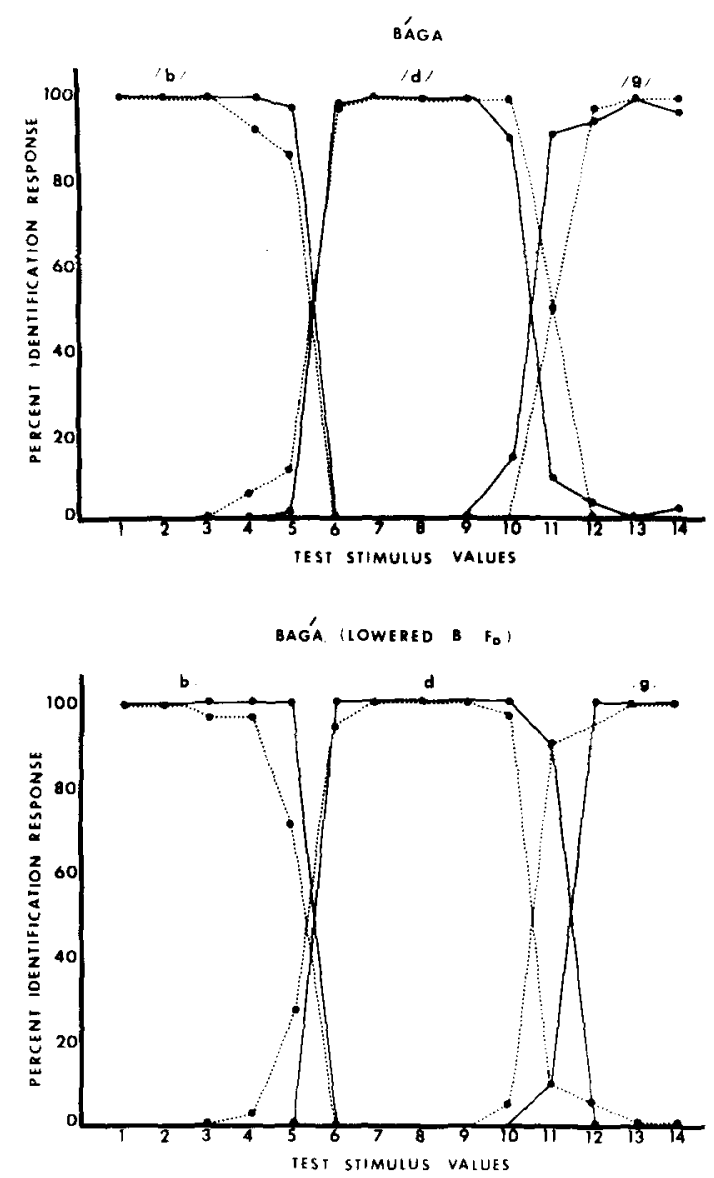

Figure 4. See caption for Figure 3.

adapting stimulus $[\mathrm{ba}][\mathrm{F}(1,8)=5.6, \mathrm{p}<.01]$ and highly significant $[\mathrm{g}]$ adaptation for the adapting stimulus [ga] $[F(1,8)=7.5, p<.01]$. In contrast, the adapting stimulus [ba] had no appreciable effect on the locus of the $[\mathrm{d}-\mathrm{g}]$ phonetic boundary $(F=.27)$ and [ga] had no effect on the locus of the [b-d] phonetic boundary $(F=.75)$.

As Table 2 shows, of the five other adapting stimuli, only [g]-adaptation for the adapting stimulus [bagá] was found to be significant $[F(1,8)=3.94$, $\mathrm{p}<.05$ ]. [B]-adaptation for this stimulus was not significant $(F=1.07)$. All other comparisons were nonsignificant $(p>.05)$. In sum, all of the initial consonants and four of the five medial consonants

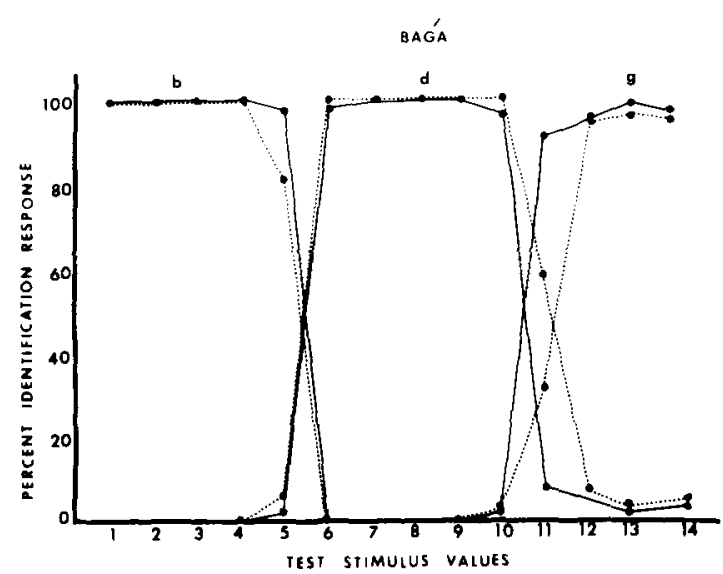

in the two-syllable adapting stimulus $\left(\mathrm{C}_{1} \mathrm{VC}_{2} \mathrm{~V}\right)$ failed to effect the perception of a $\mathrm{CV}$ [ba da ga] place of articulation continuum. These results suggest the presence of a speech processor sensitive to the acoustic characteristics of a stimulus, i.e., its syllable structure and organization, rather than merely its phonetic structure.

\section{DISCUSSION}

The present study was undertaken to investigate the effects of syllabic stress and segment structure on adaptation. To this end, a CV place of articulation test continuum was selectively adapted by seven adapting stimuli. Two of these stimuli, [ba] and [ga], were identical to the 1st and 12th stimuli of the test series. The other five adapting stimuli were of $C_{1} V_{2} V$ construction with either equal stress placed on the two syllables or one stressed and one unstressed syllable. As demonstrated in several previous studies (e.g., Blumstein, Stevens, \& Nigro, 1977; Cooper, 1974a), the two adapting stimuli [ba] and [ga] effected significant shifts in the locus of the phonetic boundary toward the adapting phonetic category. Of the five twosyllable adapting stimuli, only [bagá] produced significant identification boundary shifts. However, this shift was observed for only the [d-g] phonetic boundary ([g]-adaptation) and not the [b-d] boundary ([b]-adaptation). Thus, results for the

Table 2

Tests of Significance for Shifts in Phonetic Boundary Loci Between the Unadapted Test Condition and Each Adaptation Condition

\begin{tabular}{|c|c|c|c|c|c|c|c|c|c|c|c|c|c|c|}
\hline & \multicolumn{7}{|c|}{ [b-d] Phonetic Boundary } & \multicolumn{7}{|c|}{ [d-g] Phonetic Boundary } \\
\hline & [ba] & [ga] & $\begin{array}{r}\text { Ad } \\
\text { [baga] }\end{array}$ & $\begin{array}{c}\text { aptation } \\
\text { [gaba] }\end{array}$ & $\begin{array}{l}\text { with } \\
\text { [bága] }\end{array}$ & [bagá] & [bagá] * & [ba] & [ga ] & $\begin{array}{l}\text { Ada } \\
\text { [baga] }\end{array}$ & $\begin{array}{c}\text { ptation } \\
\text { [baga] }\end{array}$ & $\begin{array}{l}\text { with } \\
\text { [bága] }\end{array}$ & [bagá] & [bagá]* \\
\hline F Value & $5.60 \dagger$ & .27 & 2.60 & .60 & 1.20 & 1.07 & -1.27 & .75 & $7.50 \dagger$ & 2.80 & .63 & 2.13 & $3.94+t$ & .63 \\
\hline
\end{tabular}

Note-All $F$ values are nonsignificant uniess otherwise indicated.

* Lowered lbal fundamental frequency. $t_{p}<.01 \quad$ t $p<.05$ 
initial consonants in all of the two-syllable adapting stimuli $\left(\mathrm{C}_{1} \vee \mathrm{C}_{2} \mathrm{~V}\right)$ failed to affect the perception of a $C V$ place of articulation continuum, while the medial consonants affected adaptation in only one of the five conditions.

Ades (1974) demonstrated that the adapting stimuli [b] and [d] in final position failed to fatigue the detectors responsible for [b] and [d] in initial position, and vice versa. These results were interpreted as evidence against a phonetic speech processor since such a detector mechanism would not distinguish between initial and final syllable environments, but would be sensitive only to the phonetic characteristics of the stimulus independent of its position. Results of the present study also argue against a phonetic processor, as consonants in medial position of all but one of the adapting conditions failed to produce significant adaptation of acoustically identical consonants in the initial position of the test stimuli (i.e., the adapting stimulus [baga] failed to produce significant $[\mathrm{g}]$-adaptation). ${ }^{1}$ The adapting stimulus [bagá] did produce significant adaptation for the medial consonant. One might argue that stress, as conditioned by vowel length and fundamental frequency, may have affected adaptation in this condition. However, as only one of the adapting stimuli with primary stress on the second syllable showed significant adaptation effects, stress placement per se cannot be a conditioning factor. It may well be that the acoustic cues used to vary stress have differential perceptual weights. It is noteworthy in this regard that significant effects were obtained when both fundamental frequency and vowel length were varied, but not when only fundamental frequency was varied.

Although stress placement proved not to be a significant variable, the acoustic properties which signal this phonetic dimension. (i.e., fundamental frequency and duration) may, by themselves, contribute to the obtained adaptation effects. In the stimulus [bagá], the fundamental frequency of the stressed second syllable was raised but the syllabic length was kept the same. Fundamental-frequency characteristics of a syllable do not seem to have a systematic effect on adaptation. In particular, raising the fundamental frequency in the initial syllable of the stimulus [bága] failed to demonstrate [b]-adaptation. It has also been demonstrated (Blumstein, Note 1) that changing the fundamental frequency of the adapting stimulus has no differential effect on adaptation. In fact, adaptation has been shown for a CV synthetic continuum, when the adapting stimulus was a natural speech exemplar differing not only in onset frequencies but also fundamental frequency (Cooper \& Blumstein, 1974). Similarly, syllable duration does not seem to be a relevant dimension. Although adaptation effects were obtained for the medial consonant [g] when the syllable duration of the adapting stimulus [ga] was of the same length as the test stimulus [ga], adaptation effects were not obtained for the initial consonant when the initial vowel was either shortened or of the same duration as that of the test stimulus. That is, both [bagá] and [bága] failed to evidence [b]-adaptation.

Ades (1974) has postulated the existence of a different set of feature detectors for consonants in initial and final position. Given the results of the present study, this theory may be extended to suggest the existence of detectors for consonants in medial position as well. Thus, effects of adaptation are conditioned only in part by phonetic structure. In addition, findings for the present study argue strongly against a speech processor sensitive only to rising vs. falling transitions as was originally suggested by Ades. If this were the case, adaptation effects would have been obtained for the stimuli in intervocalic position since the acoustic properties (e.g., onset frequencies and formant transitions) were identical to the stimuli of the test continuum.

The most surprising result of this study is that the initial syllable of the two-syllable adapting stimuli failed in all conditions to significantly adapt a continuum of one-syllable CV stimuli. The consonants in the adapting and test stimuli were in absolute-initial as wel as syllable-initial position. Thus, not only were the phonetic properties of the initial segments identical, but also the onset frequency characteristics and overall shape of the spectrum at onset were equivalent (see Footnote 1). These findings suggest that the operation of detector mechanisms susceptible to fatigue by an adapting stimulus are even more constrained than has heretofore been suggested. It appears that the adapting and test stimuli must have not only the same phonetic and syllable structure but also the same syllable organization (i.e., the same number of syllables) for the adaptation effect to occur.

Another possible, if less interesting, explanation for the present findings involves an interaction, possibly in the form of masking, that is occurring between the consonants in each syllable. That is, the [ba] of either the first or second syllable in the stimulus may mask or interfere with the perception of [ga] of the other syllable. In this case, no adaptation effects would be found.

In contrast to the results obtained in this study, Rudnicky and Cole (1977) report significant adaptation effects for initial consonants when the adapting stimuli were presented using connected speech. In this case, the syllable structures of adapting and test stimuli were not identical, and yet, significant effects were obtained. It is difficult to 
compare the results of that study with the present study as they differed in several ways. Rudnicky and Cole investigated adaptation effects for voicing and manner of articulation, not place of articulation. The stimulus continua they used consisted of smaller acoustic steps. Finally, the adapting stimuli used in connected discourse were not all of equal duration and they were not presented at a completely uniform rate.

Further research is clearly needed to investigate the limitations of the adaptation effect in speech perception. What the present study suggests is that adaptation effects may occur at a very low level of speech analysis constrained by such characteristics of the speech signal as its acoustic attributes and phonetic quality as well as its syllable and segment structure.

\section{REFERENCE NOTE}

1. Blumstein, S. E. Personal communication, April 10, 1977.

\section{REFERENCES}

ADEs, A. E. How phonetic is selective adaptation? Experiments on syllable position and vowel environment. Perception \& Psychophysics, 1974, 16, 61-67.

Blumstein, S. E., Stevens, K. N., \& Nigro, G. N. Property detectors for bursts and transitions in speech perception. Journal of the Acoustical Society of America, 1977, 61, 1301-1313.

CoOper, W. E. Adaptation of phonetic feature analyzers for place of articulation. Journal of the Acoustical Society of America, 1974, 56, 617-627, (a)

COOPER, W. E. Contingent feature analysis in speech perception. Perception \& Psychophysics, 1974, 16, 201-204. (b)

Cooper, W. E., \& Blumstein, S. E. A 'labial' feature analyzer in speech perception. Perception \& Psychophysics, 1974, 15, 591-600.

DIEHL, R. H. The effect of selective adaptation on the identification of speech sounds. Perception \& Psychophysics, 1975, 17, * 48-52.

Ermas, P. D., \& Corbit, J. D. Selective adaptation of linguistic feature detectors. Cognitive Psychology, 1973, 4, 99-109.

Evans, E. F., \& Whitfield, I. C. Classification of unit responses in the auditory cortex of the unanesthetized and unrestrained cat. Journal of Physiology. 1964. 171, 476-493.

Fry. D. B. Experiments in the perception of stress. Language and Speech, 1958, 1, 126-152.

Greenberg, J. J., \& Jenkins, J. J. Studies in the psychological correlates to the sound system of English. Word, 1964, 20, 157.177 .

KLATt, D. Acoustical theory of terminal analog speech synthesis. In Proceedings of the 1972 Intermational Conference of Communication and Processing, 1972. Boston.

Lehiste, 1., \& Peterson, G. E. Vowel amplitude and phonemic stress in American English. Journal of the Acoustical Society of America, 1959, 31, 428-435.

Miller, G., \& Nicely, P. E. An analysis of pereeptual confusions among English consonants. Joumal of the Acoustical Society of America. 1955, 27, 338-352.

Pisoni, D. B.. \& TASH, J. Auditory property detectors and processing place features in stop consonants. Perception \& Psychophysics, 1975, 18, 401-408.

Rudnicky, A., \& Cole, R. A. Adaptation produced by connected speech. Journal of Experimental Psychology: Human Perception and Performance, 1977, 3, 51-61.

SingH, S. Cross-language study of perceptual confusions of plosive phonemes in two conditions of distortion. Joumal of the Acoustical Society of America, 1966, 40, 635-656.

TartTer, V. C., \& Eimas, P. D. The role of auditory feature detectors in the perception of speech. Perception \& Psychophysics, 1975, 18, 293-298.

WICKELGREN, W. Acoustic similarity and intrusion errors in shortterm memory. Journal of Experimental Psychology. 1965, 70, 102-108.

WiCKElGREN, W. Distinctive features and error in short-term memory for English consonants. Journal of the Acoustical Society of America, 1966, 39, 388-398.

\section{NOTE}

1. Although intervocalic consonants in natural speech may have an additional cue of closure duration to signal place of articulation, in the present study closure duration was not included in the parameters of the stimuli. As described in the stimulus section, the stimuli consisted of two CV syllables separated by $80 \mathrm{msec}$ of silence. There were no consonantal offsets on the $V$ preceding the intervocalic consonant, and consequently, there was no acoustic cue of closure duration present in these stimuli. The onset characteristics of the [B] in [ga] and [baga] then were acoustically identical.

(Received for publication June 23, 1977; revision accepted April 14, 1978.) 